\title{
ARTICLE
}

Received 28 Oct 2012 | Accepted 16 May 2013 | Published 18 Jun $2013 \quad$ DOl: 10.1038/ncomms3017

\section{Spin readout and addressability of phosphorus-donor clusters in silicon}

\author{
H. Büch ${ }^{1}$, S. Mahapatra ${ }^{1, \dagger}$, R. Rahman ${ }^{2, \dagger}$, A. Morello ${ }^{1} \&$ M.Y. Simmons ${ }^{1}$
}

The spin states of an electron bound to a single phosphorus donor in silicon show remarkably long coherence and relaxation times, which makes them promising building blocks for the realization of a solid-state quantum computer. Here we demonstrate, by high-fidelity (93\%) electrical spin readout, that a long relaxation time $T_{1}$ of about $2 \mathrm{~s}$, at $B=1.2 \mathrm{~T}$ and $T \approx 100 \mathrm{mK}$, is also characteristic of electronic spin states associated with a cluster of few phosphorus donors, suggesting their suitability as hosts for spin qubits. Owing to the difference in the hyperfine coupling, electronic spin transitions of such clusters can be sufficiently distinct from those of a single phosphorus donor. Our atomistic tight-binding calculations reveal that when neighbouring qubits are hosted by a single phosphorus atom and a cluster of two phosphorus donors, the difference in their electron spin resonance frequencies allows qubit rotations with error rates $\approx 10^{-4}$. These results provide a new approach to achieving individual qubit addressability.

\footnotetext{
${ }^{1}$ Australian Research Council Centre of Excellence for Quantum Computation and Communication Technology, University of New South Wales, Sydney, New South Wales 2052, Australia. ${ }^{2}$ Advanced Device Technologies, Sandia National Laboratories, Albuquerque, New Mexico 87185, USA. †Present addresses: Department of Physics, Indian Institute of Technology Bombay, Powai, Mumbai 400076, India (S.M.); Network for Computational Nanotechnology, Purdue University, Indiana 47906, USA (R.R.). Correspondence and requests for materials should be addressed to M.Y.S. (email: michelle.simmons@unsw.edu.au).
} 
S ilicon is an attractive material to host spin qubits because of its low spin-orbit coupling and natural abundance of zero nuclear spin isotopes, leading to long decoherence $\left(T_{2}\right)$ (refs 1-3) and relaxation times $\left(T_{1}\right)$ (refs 4-6). Particularly, in bulk P-doped $\mathrm{Si}(\mathrm{Si}: \mathrm{P})$, a remarkably long $T_{1}$ was measured $(\approx 3,000 \mathrm{~s}$ at $T=1.3 \mathrm{~K}$ and $B=320 \mathrm{mT})$ in ref. 4 . Recent results have demonstrated high-fidelity electron spin readout of a single implanted $\mathrm{P}$ donor in silicon with $T_{1}=6 \mathrm{~s}$ at $T=100 \mathrm{mK}$ and $B=1.5 \mathrm{~T}$ (ref. 6), thereby establishing one of the key milestones of realizing a scalable spin quantum computing architecture ${ }^{7-9}$. Here, we demonstrate that long $T_{1}$ is retained also for electronic spin states bound to an ultra-small cluster of $\mathrm{P}$ donors, precisely engineered by scanning tunnelling microscope (STM) hydrogen lithography.

\section{Results}

STM-patterned device layout. The spin readout architecture used in this study consists of an ultra-small donor cluster $(\approx 4 \mathrm{P}$ donors), both tunnel and capacitively coupled to an adjacent single electron transistor (SET) (Fig. 1a,b). Exploiting the atomicscale precision of STM hydrogen lithography, this planar architecture was fabricated and previously demonstrated to exhibit high contrast charge-sensing characteristics ${ }^{10}$. To review the device layout, Fig. 1b shows a filled-state STM image, where the yellow features correspond to the device template, generated by desorbing the hydrogen resist monolayer (blue) with the STM tip. Subsequent exposure of the $\mathrm{Si}$ surface to $\mathrm{PH}_{3}$ gas and annealing incorporate $\mathrm{P}$ donors, only within the device pattern ${ }^{10}$. A closeup of the central part of the device, after the lithography step, is seen in Fig. 1a. The two terminals source (S) and drain (D), along with the island $D_{1}$, define the SET. The pattern which creates the P-donor cluster $\left(D_{2}\right.$ in Fig. 1a) is situated at a distance of $16 \mathrm{~nm}$ from $D_{1}$. Two gates $G_{1}$ and $G_{2}$ are optimally situated (Fig. $1 b$ ) to gain sufficient differential control of the electrochemical potentials of $\mathrm{D}_{1}$ and $\mathrm{D}_{2}\left(\mu_{\mathrm{D} 1}\right.$ and $\left.\mu_{\mathrm{D} 2}\right)$.

The lithographic template of $\mathrm{D}_{2}$ (Fig. 1c) reveals two rows of five adjacent Si dimers (outlined with green ellipses in Fig. 1d,e), which can accommodate a maximum of four $\mathrm{P}$ donors (see Supplementary Fig. S1 and Supplementary Methods) ${ }^{11}$. Two possible configurations of these four donors are shown by the white circles. Being closely packed within an area of $1.5 \mathrm{~nm} \times 2 \mathrm{~nm}$, the largest possible separation between the donors is $\approx 2 \mathrm{~nm}$ (Fig. 1d), which is smaller than the Bohr radius of a single $\mathrm{P}$ donor in $\mathrm{Si}\left(a_{\mathrm{B}}=2.5 \mathrm{~nm}\right)$. Therefore, the donor cluster $D_{2}$ behaves as a single quantum dot. $D_{2}$ is tunnel coupled to $D_{1}$, so that charging and discharging of $D_{2}$ occur by exchanging electrons with $D_{1}$, eliminating the need for any additional electron reservoir.

Change in the charge state of $\mathrm{D}_{2}$ results in a drastic change of the SET conductance. This is revealed in Fig. 1f, where the SET current is mapped as a function of $V_{\mathrm{G} 1}$ and $V_{\mathrm{G} 2}$ for a fixed source-drain bias $\left(V_{\mathrm{SD}}=0.5 \mathrm{mV}\right)$. Each line corresponds to charge transitions of $\mathrm{D}_{1}$ and exhibits discrete breaks, which reflects a shift of the SET bias point in capacitive response to charging and discharging of $\mathrm{D}_{2}$. Three such lines of breaks, denoted by A, B and C in Fig. 1f, correspond to three charge transitions of $\mathrm{D}_{2}$. A total of seven charge transitions could be identified for $\mathrm{D}_{2}$ in a larger-area stability map ${ }^{10}$, but we saw no evidence of completely depleting the donor cluster within our available gate space.

To quantify the sensitivity of charge detection, Fig. 1g shows $V_{\mathrm{G} 1}$ projections of the stability map of Fig. 1f, for two different fixed values of $V_{\mathrm{G} 2}$ (dotted and solid vertical lines in Fig. 1f). These were chosen from either side of a particular charge transition line of $\mathrm{D}_{2}$ (denoted by $\mathrm{B}$ ) and have been superimposed
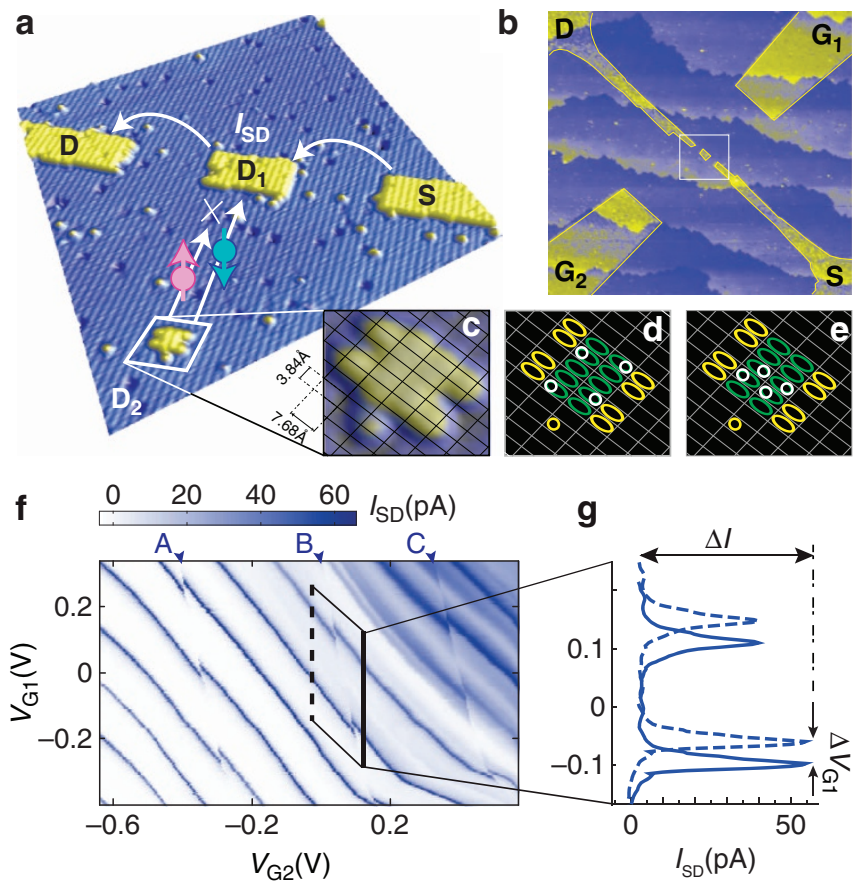

Figure 1 | Spin readout architecture for donor clusters. (a) Central part of the device (in yellow), patterned by desorbing the hydrogen mask (in blue), showing the SET island $\left(D_{1}\right)$, the few-donor quantum dot $\left(D_{2}\right)$, and the innermost sections of the source and the drain leads of the SET. Spindependent tunnelling between $D_{1}$ and $D_{2}$ is shown schematically. (b) The overall device, showing the layout of the two gates $\left(G_{1}, G_{2}\right)$ and the extensions of the $S$ and $D$ leads. The white box shows the area shown in $\mathbf{a}$. (c) Close-up image of $D_{2}$, overlaid by a grid with dimer-row spacing, revealing the exposed silicon dimers. (d,e) Schematic representation of $D_{2}$, showing sites suitable for $\mathrm{P}$ donor incorporation in green and the rest in yellow. The set of four white circles show two possible configurations of the $P$ donors. (f) Map of SET current $\left(I_{\mathrm{SD}}\right)$ versus gate voltages $V_{\mathrm{G} 1}, V_{\mathrm{G} 2}$, at an applied bias of $V_{S D}=0.5 \mathrm{mV}$, showing breaks in $I_{S D}$ due to charging of $D_{2}$. (g) $V_{\mathrm{G} 1}$ projections of $I_{\mathrm{SD}}$, corresponding to the solid and dashed lines in $\mathbf{f}$. The shift of the SET bias point due to charging of $D_{2}$ and the current contrast due to charge sensing are indicated by $\Delta V_{\mathrm{G} 1}$ and $\Delta l$, respectively.

on each other. The shift in the SET peak position $\left(\Delta V_{\mathrm{G} 1}=28\right.$ $\mathrm{meV}$ ) due to charging of $\mathrm{D}_{2}$ is large enough for the SET current to switch from its maximum to almost its minimum value $(\Delta I)$, revealing nearly $100 \%$ contrast in charge sensing. This is an inherent advantage of charge sensing with a SET sensor, which enables high-fidelity spin readout ${ }^{6,12}$.

Single-shot spin readout. Having established the suitability of a STM-patterned SET for high contrast charge sensing ${ }^{10}$, we now demonstrate single-shot spin readout in this architecture, following the protocol initially developed by Elzerman et al. ${ }^{13}$ This technique depends on spin-to-charge conversion by application of a magnetic field $(B)$ and tracking in real time the current response of the SET to a three-level gate voltage pulse which loads, reads, and empties the up $(\mid \uparrow>)$ and down $(\mid \downarrow>)$ spin states of $\mathrm{D}_{2}\left(\mu_{\mathrm{D} 2}^{\uparrow}\right.$ and $\mu_{\mathrm{D} 2}^{\downarrow}$, respectively). We apply a threelevel gate voltage pulse at the transition point shown in Fig. 2a (line B in Fig. 1f), with the blue, brown and orange points representing the load, read, and empty positions, respectively. In the load phase, both $\mu_{\mathrm{D} 2}^{\uparrow}$ and $\mu_{\mathrm{D} 2}^{\downarrow}$ are pulled below $\mu_{\mathrm{D} 1}$, whereby an electron of either spin-state tunnels onto $\mathrm{D}_{2}$, after a characteristic tunnelling time, $\tau_{\text {load. }}$. This switches the SET 'off and is seen in the single-shot current response as a sudden drop 
a

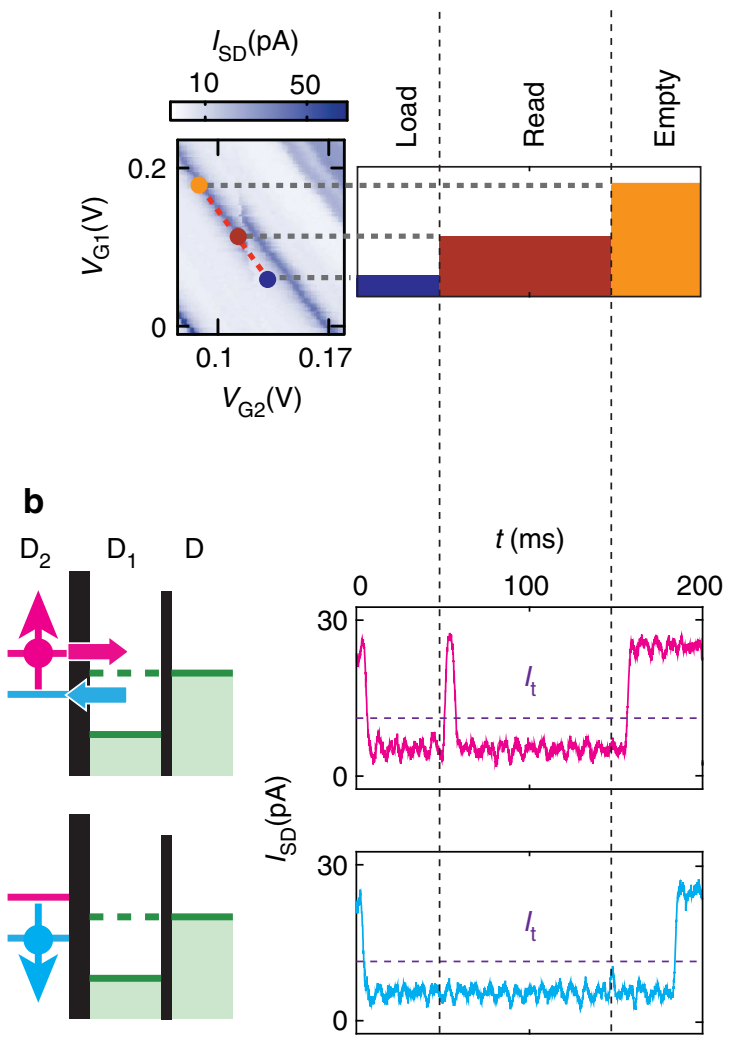

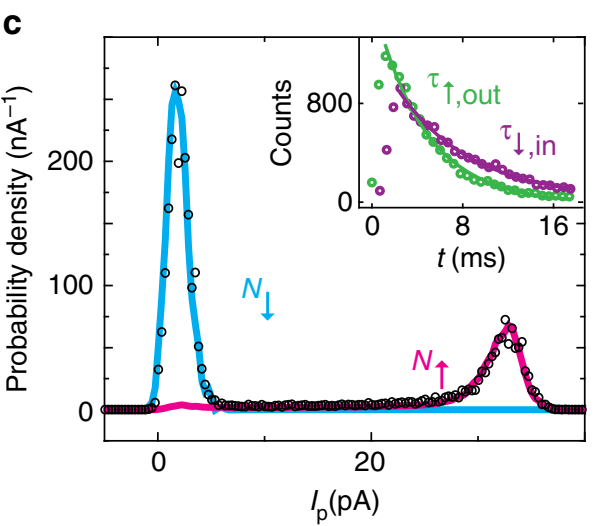

d

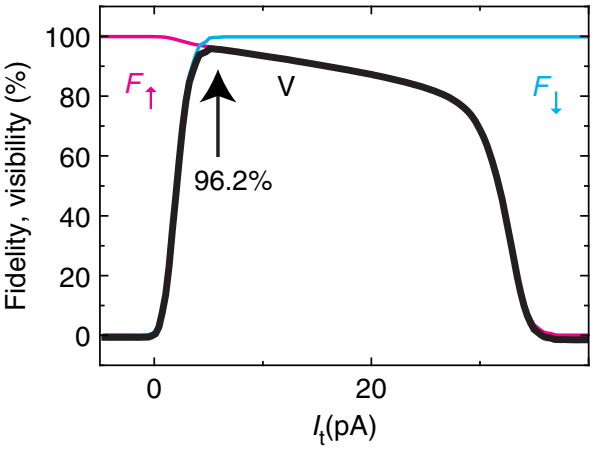

Figure 2 | High-fidelity single-shot spin readout. (a) A section of the stability plot of the SET and the applied three-level gate voltage pulse for single-shot spin readout. (b) Spin-dependent SET current response, showing a current peak for $\mid \uparrow>$ (top) and no peak for $\mid \downarrow>$ (bottom) detection. (c) Histogram of the current response maxima $\left(I_{p}\right)$ for a fixed time interval $(\Delta t=40 \mathrm{~ms})$ in the readout phase, revealing two well-separated peaks. This distribution is fitted with the sum of two calculated histograms $N_{\uparrow}$ (pink) and $N_{\downarrow}$ (blue). Inset: histogram of the tunnel-out times of the | $\uparrow>$ (green) and subsequent tunnel-in times of the $\mid \downarrow>$ (purple) electrons. Exponential fits to the histograms provide the corresponding tunnel rates, necessary for calculation of $N_{\uparrow}$ and $N_{\downarrow}$. (d) Readout fidelities $\left(F_{\uparrow}\right.$ and $\left.F_{\downarrow}\right)$ and visibility $(V)$ as a function of threshold current, $I_{\mathrm{t}}$.

of $I_{\mathrm{SD}}$ to its minimum value (see load columns in Fig. 2a,b). In the read phase, the pulse amplitude is so chosen that

$$
\mu_{\mathrm{D} 2}^{\downarrow}<\mu_{\mathrm{D} 1}<\mu_{\mathrm{D} 2}^{\uparrow} .
$$

This is shown in the schematic illustrations of Fig. 2b. Here, $I_{\mathrm{SD}}$ remains a minimum if a $\mid \downarrow>$ electron is loaded (see read column of lower panel in Fig. 2b). On the other hand, if an $\mid \uparrow>$ electron is loaded, it tunnels back to $D_{1}$ and subsequently, a $\mid \downarrow>$ electron loads onto $\mathrm{D}_{2}$. This results in a peak in the SET current response (read column of upper panel in Fig. 2b), as the SET is switched 'on' for a short while. In the final empty phase, $\mathrm{D}_{2}$ is unloaded regardless of the spin state by plunging both $\mu_{\mathrm{D} 2}^{\uparrow}$ and $\mu_{\mathrm{D} 2}^{\downarrow}$ above $\mu_{\mathrm{D} 1}$. This switches the SET 'on', until the next load phase starts.

An isolated spike of the SET current in the read phase thus serves as an evidence of a $\mid \uparrow>$ electron being detected in this single-shot electrical spin readout scheme. For quantum computing applications, it is important to assess the fidelity of this detection process, that is, the probability of identifying the electronic spin state correctly from the current response of the SET. To quantify spin readout fidelity, we investigate the peak values of the SET current $\left(I_{p}\right)$, recorded over a certain time interval $\Delta t=40 \mathrm{~ms}$ in the read phase, for 20,000 traces. The distribution of $I_{\mathrm{p}}$ is shown by the open circles in Fig. 2c, where the peak at high (low) $I_{\mathrm{p}}$ is consistent with the current response of the SET for detection of the $\mid \uparrow>(\mid \downarrow>)$ state. This distribution represents the sum $N=N_{\downarrow}+N_{\uparrow}$, where $N_{\uparrow}$ and $N_{\downarrow}$ are the separate histograms for each spin configuration. $N_{\uparrow(\downarrow)}$ cannot be deduced experimentally, but can be obtained by numerical modelling ${ }^{6}$, knowing the peak height $(\Delta I)$, the detection bandwidth $(300 \mathrm{~Hz})$ and characteristic times for $\mid \uparrow>-(\mid \downarrow>)$ electron tunnelling out of (onto) $\mathrm{D}_{2}, \tau_{\uparrow \text {,out }}\left(\tau_{\downarrow, \text { in }}\right)$. Values of $\tau_{\uparrow \text {,out }}=3.9 \mathrm{~ms}$ and $\tau_{\downarrow \text {,in }}=6.9 \mathrm{~ms}$ were obtained from exponential fits to the histograms of the peak position and width, respectively, as shown in the inset of Fig. 2c. The calculated histograms for $N_{\uparrow}$ (pink) and $N_{\downarrow}$ (blue) sum up to match very well with the recorded distribution of $I_{\mathrm{p}}$ in Fig. 2c. From the $N_{\uparrow(\downarrow)}$ histograms, the readout fidelities of the $\mid \uparrow>$ and $\mid \downarrow>$ spin states, $F_{\uparrow}$ and $F_{\downarrow}$, as well as the readout visibility, defined as $V=F_{\downarrow}+F_{\uparrow}-1$, can be determined as a function of threshold current $I_{\mathrm{t}}$ and are shown in Fig. 2d. As evident in the plot, the visibility reaches a maximum of $V=96.2 \%$ at an optimum threshold current of $I_{\mathrm{t}}=5.4 \mathrm{pA}$, where $F_{\uparrow}=96.4 \%$ and $F_{\downarrow}=99.8 \%$. However, a more rigorous estimate of visibility, taking spin-to-charge conversion losses into account, leads to $V=93.4 \%$ (details in Supplementary Fig. S2 and Supplementary Note 1).

Determining $T_{1}$ in P-donor clusters. Having established the feasibility of discriminating the spin states with sufficiently high fidelity, we now turn to measurement of $T_{1}$. To determine $T_{1}$, single-shot current response of the SET is monitored for varying lengths of the load pulse $t_{\text {load }}$. As $t_{\text {load }}$ is made longer, the fraction of $\mid \uparrow>$ detected in the readout phase decays exponentially, due to relaxation to the ground $\mid \downarrow>$ state. Figure 3 a shows this decay 

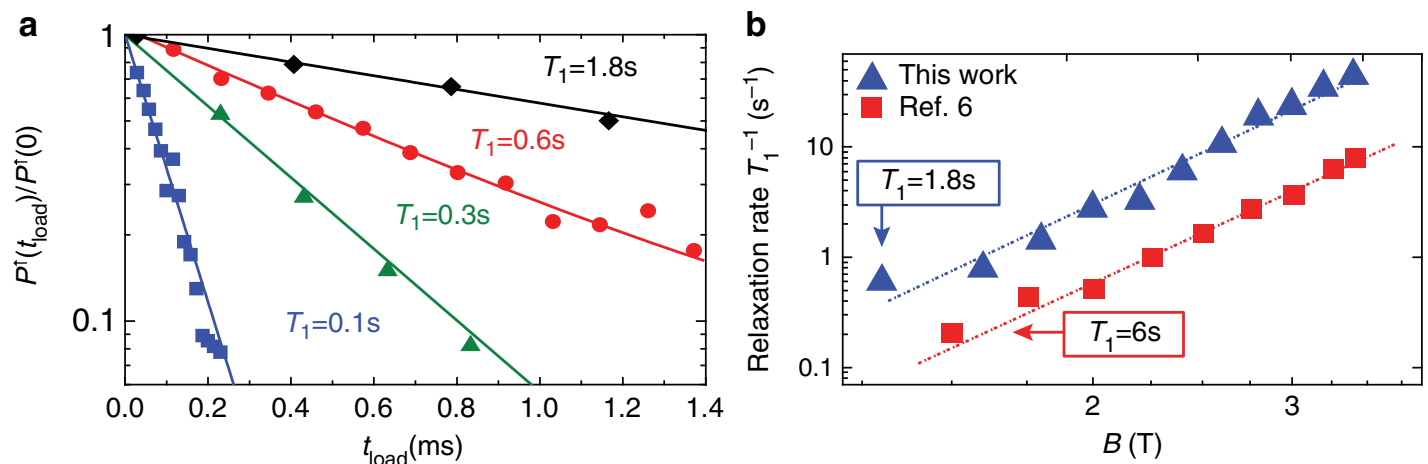

Figure 3 | Spin-lattice relaxation time of a $\mathbf{P}$ donor cluster. (a) Normalized $\mid \uparrow>$ fraction as a function of $t_{\text {load, }}$ for different $B$-fields from $1.2 T$ (black diamonds) in steps of 0.4-2.6 T (blue squares). Exponential fit to the data yields $T_{1}$. (b) $B$-field dependence of $T_{1}^{-1}$ of the device, together with that of an implanted donor (data from ref. 6).

of the $\mid \uparrow>$ fraction at different magnetic fields. An exponential fit to the data yields $T_{1}$, which for $B=1.2 \mathrm{~T}$ is $\approx 1.8 \mathrm{~s}$. Plotting the decay rates at different magnetic fields, we find a $B^{5}$ dependence of $T_{1}^{-1}$, as shown by the blue triangles in Fig. 3b. For comparison, we plot in the same figure, similar data for a single implanted $\mathrm{P}$ donor (reproduced from ref. 6), wherein the spin-lattice relaxation time was found to follow $T_{1}^{-1}=K_{5} B^{5}$, with $K_{5}=0.015 \mathrm{~T}^{-1} \mathrm{~s}^{-1}$. The $B^{5}$ dependence of $T_{1}^{-1}$ reflects spin relaxation via the acoustic-phonon-mediated valley repopulation mechanism $^{14}$, at the limit $k_{\mathrm{B}} T \ll g_{\mathrm{e}} \mu_{\mathrm{B}} B$ (ref. 6). Our data therefore strongly suggest that the same mechanism as for the case of single $\mathrm{P}$ donors in $\mathrm{Si}$ is also responsible for relaxation of the many-electron spin excited state, hosted by the P-donor cluster.

Single-shot spin readout was also attempted at a few other triple points. For other transitions along line B (same charge state of $\mathrm{D}_{2}$ ), we measured similar values of $T_{1}$. However, spin readout could not be performed for transitions on line A (line $\mathrm{C}$ ), which represents one electron less (more) on $\mathrm{D}_{2}$, which is consistent with pairwise spin filling.

Further insight on the absolute values of $T_{1}$ can be obtained by analysing the coefficient $K_{5}$, which is an order of magnitude higher $\left(K_{5}=0.134 \mathrm{~T}^{-1} \mathrm{~s}^{-1}\right)$ in our case. All parameters in $K_{5}$ are related to the the silicon bulk ${ }^{6,14}$ except for the valley-orbit splitting $\left(\Delta E_{\mathrm{V}}\right)$, which is sensitive to the nature of confinement potential, and hence to the number and distribution of the $\mathrm{P}$ donors defining the quantum dot. $K_{5}$ scales $\propto \Delta E_{\mathrm{V}}^{-2}$, which suggests that for the P-donor cluster $\Delta E_{\mathrm{V}} \approx 3.5 \mathrm{meV}$. Interestingly, a very similar valley-orbit splitting has been observed for an exciton bound to a P in Si (ref. 15). Although an exact estimate of the valley-orbit splitting is computationally expensive for the system studied here, the lower value observed (compared with single $\mathrm{P}$ donors $\left(\Delta E_{\mathrm{V}}^{\text {bulk }}=10.6 \pm 0.2 \mathrm{meV}\right.$ (ref. 16)) can be attributed to screening and correlation effects in a multi-electron quantum dot. A larger $\Delta E_{\mathrm{V}}$, and hence a longer $T_{1}$, will therefore be expected for the spin excited state of the last electron bound to the same quantum dot.

Donor clusters as hosts for single-spin qubits. The above results indicate that a long spin-lattice relaxation time can be retained even when single electronic spins are confined to small P-donor clusters. This fact opens up the prospect of using donor clusters as hosts for single-spin qubits and can be of significant consequence in performing single qubit rotations in a large array of Si:P qubits, as required for a practical quantum computing architecture. A daunting challenge of performing single qubit rotations with qubit arrays hosted by individual $\mathrm{P}$ donors is to excite electron spin resonance (ESR) transitions of a particular qubit, without affecting neighbouring ones. To achieve this selectivity, tuning ESR transitions of a targeted qubit into resonance with a globally applied microwave field (typically $30-40 \mathrm{GHz}$ ), by electrical control of the hyperfine coupling between the electron- and the donor-nuclear spins, has been proposed ${ }^{17}$. The ESR frequency of a single-P-donor qubit in a static magnetic field $B$ is given by

$$
v=\frac{1}{h}\left(g_{\mathrm{e}}(E) \mu_{\mathrm{B}} B \pm \frac{A(E)}{2}+\frac{A(E)^{2}}{\left(g_{\mathrm{e}}(E) \mu_{\mathrm{B}}-g_{\mathrm{n}} \mu_{\mathrm{n}}\right) B}\right)
$$

where $h$ is the Planck's constant; $\mu_{\mathrm{n}}=e / 2 m_{\mathrm{n}}$, the nuclear magneton; $g_{\mathrm{n}}$, the nuclear gyromagnetic ratio; and $A(E)$ and $g_{\mathrm{e}}(E)$, the electric field $(E)$ tunable hyperfine coupling and electron gyromagnetic ratio, respectively. Here, we have included the $E$-field dependence of the $g$ factor, which was ignored in previous donor spin qubit proposals ${ }^{17}$, but was measured in ESR experiments $^{18}$. The selectivity of single qubit rotation in the canonical scheme relies on Stark shift of $A(E)$ only to bring $v$ in resonance with the microwave field $\left(v_{\mathrm{MW}}\right)$, while all others qubits stay sufficiently detuned. However, the Stark tunability of $A(E)$ $\left(\approx 0.4 \mathrm{MHz} /(\mathrm{MV} / \mathrm{m})^{2}\right)$ and of the spin-orbit interaction $\mu_{\mathrm{B}} g_{\mathrm{e}}(E)$ $\left(\approx 0.3 \mathrm{MHz} /\left(\mathrm{T}(\mathrm{MV} / \mathrm{m})^{2}\right)\right)$ may be insufficient to achieve the frequency shifts necessary for a required single qubit rotation fidelity $\left(>10^{-5}\right)$ in realistic $E$-fields $\left(\leq 10 \mathrm{MV} \mathrm{m}^{-1}\right)$.

\section{Discussion}

On the basis of atomistic tight-binding modelling, in this section, we discuss that by hosting consecutive single-spin qubits in an array of clusters with different number of P donors, a 'built-in' selectivity of ESR frequencies can be achieved naturally. This arises due to the extreme sensitivity of the hyperfine coupling to the number and distribution of nuclear spins (see Supplementary Fig. S3). To demonstrate this, we plot in Fig. 4a the E-field (applied along the [1ㅣㅣㄹ direction) dependence of the ESR transition frequencies of both a single-donor (red lines) and a 2-P-donor cluster (blue lines), in a static magnetic field $B=1.5 \mathrm{~T}$. Such a two donor cluster (shown in the inset) can be realised by STM patterning (see Methods). The four blue lines in the plot correspond to the four possible ESR transitions between the eight eigenstates of the three-spin (one electron and two $\mathrm{P}$ nuclei) system, obtained by solving the effective spin Hamiltonian

$$
H=g_{\mathrm{e}}(E) \mu_{\mathrm{B}} B \sigma_{\mathrm{e}}^{z}+g_{\mathrm{n}} \mu_{\mathrm{n}} B \sum_{i=1}^{2} \sigma_{\mathrm{n}, i}^{z}+\sum_{i=1}^{2} A_{i}(E) \mathbf{s}_{\mathrm{e}} \cdot \mathbf{s}_{\mathrm{n}, i}
$$

where $\mathbf{s}\left(\sigma^{z}\right)$ is the Pauli $(-Z)$ matrix, with $z$ representing the direction of the $B$-field, and e and n, $i$ referring to the electron and the two $\mathrm{P}$ nuclei, respectively. The strength of the hyperfine 

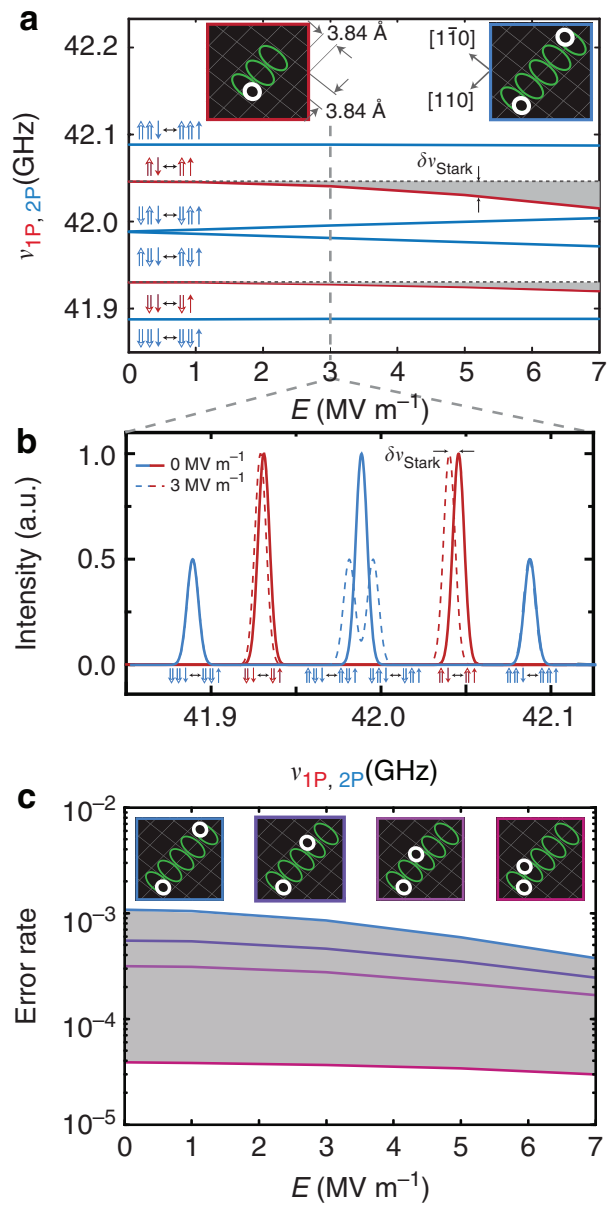

Figure 4 | ESR addressability of 1-P and 2-P qubits. (a) E-field (applied along [110]) dependence of ESR transition frequencies (blue lines) at $B=1.5$ T of the 2-P cluster (patterned along [110]), shown in the right panel of the inset. The same data for a single P donor (shown in the left panel of the inset) are shown by the red lines. (b) A schematic spectral representation of calculated resonance frequencies for 1-P and 2-P cluster at $E=0$ (solid) and $E=3 \mathrm{MVm}^{-1}$ (dashed) using a peak width of $\approx 8 \mathrm{MHz}$ (ref. 9). (c) E-field dependence of the error rate, that is, the probability of exciting an ESR transition of the 2-P qubit (1-P qubit), when the global microwave field is in resonance with a transition of the 1-P qubit (2-P qubit), for different inter-donor separations (as shown in the inset). The oscillating magnetic field, $B_{\mathrm{ac}}=10^{-4} \mathrm{~T}$, and the frequency difference correspond to the $\Uparrow \downarrow \leftrightarrow \Uparrow \uparrow$ transition of the 1-P qubit and the $\Uparrow \Uparrow \downarrow \leftrightarrow \Uparrow \Uparrow \uparrow$ transition of the 2-P qubit (see main text).

coupling between the electron and the two P nuclear spins $\left(A_{i}(E)\right)$ (ref. 19), as well as the electronic $g$ factor $\left(g_{\mathrm{e}}(E)\right)$ (ref. 20), has been calculated within the tight-binding formalism (see Methods). We found that the Stark shift of the $g$ factor is at least an order of magnitude weaker for the 2-P cluster than for 1-P (see Supplementary Note 2). The E-field dependence of the ESR transition frequencies corresponding to the single-P-donor qubit is also shown in the same plot. At $E=0$, the smallest difference in the ESR frequencies of the 1-P and the 2-P qubits, $\delta v=\left|v_{1 \mathrm{P}(\Uparrow \downarrow \leftrightarrow \Uparrow \uparrow)}-v_{2 \mathrm{P}(\Uparrow \uparrow \downarrow \leftrightarrow \Uparrow \uparrow \uparrow)}\right|$ is $\approx 40 \mathrm{MHz}$. For the entire range of the studied $E$-field, $\delta v$ is significantly larger than the Stark shift of ESR frequencies of a single $\mathrm{P}$ donor $\left(\delta v_{\text {Stark }}\right.$ in Fig. 4a). Comparison between $\delta v$ and $\delta v_{\text {Stark }}$ is illustrated in Fig. $4 \mathrm{~b}$, which schematically plots ESR transitions for a single $\mathrm{P}$ and a $2-\mathrm{P}$ cluster at $E=0$ and $3 \mathrm{MVm}^{-1}$, the latter representing an $E$-field where a bulk $\mathrm{P}$ donor is likely to ionize $\mathrm{e}^{21}$.
On the basis of the values of $\delta v$, we can estimate the error rates from the Rabi solution:

$$
P(t)=\left(\frac{g_{\mathrm{e}} \mu_{\mathrm{B}} B_{\mathrm{ac}}}{h \gamma}\right)^{2} \sin ^{2}(2 \pi \gamma t)
$$

where $\gamma=\sqrt{\left(g_{\mathrm{e}} \mu_{\mathrm{B}} B_{\mathrm{ac}} / h\right)^{2}+(\delta v)^{2}}$ and $B_{\mathrm{ac}}$ is the strength of the oscillating magnetic field due to the global microwave field. Assuming $B_{\mathrm{ac}}=10^{-4} \mathrm{~T}$, the single qubit rotation operation time for the targeted qubit, $t=1 /\left(\left.4 \gamma\right|_{\delta v=0}\right)=90 \mathrm{~ns}$. The error rate, that is, the probability of rotating the untargeted qubit (for the smallest $\delta v$, as defined above) is given by the prefactor of equation (5), and is $P \approx 10^{-3}$.

We note that this value of $P$ is the most conservative estimate of the error rate. First, we have considered an inter-donor separation, which is the largest possible in a STM pattern of five dimers. For smaller inter-donor separations, the error rate can be suppressed further. This is shown in Fig. 4c for other 2-donor configurations. Second, we have assumed that for both 1-P and 2-P qubits, the nuclear spins are initialized in their respective ground states ( $\Uparrow$ and $\Uparrow \Uparrow$ ), which is the nuclear configuration where the electron resonance frequencies are the closest. If instead the 1-P qubit was initialized in the $\Downarrow$ state, then $P$ can be as small as $10^{-5}$, as $\delta v=\left|v_{1 P(\Downarrow \downarrow \leftrightarrow \Downarrow \uparrow)}-v_{2 P(\Uparrow \Uparrow \downarrow \leftrightarrow \Uparrow \uparrow \uparrow)}\right| \approx 200$ $\mathrm{MHz}$. We note that the expected nuclear spin relaxation time $\left(T_{\ln } \approx\right.$ hours at $100 \mathrm{mK}$ ) (ref. 22) means that this nuclear configuration can be maintained for extremely long times. Therefore, hosting alternate spin qubits in 1-P and 2-P donors provides a viable route to perform high-fidelity single-qubit rotation in a globally applied microwave field. A remaining challenge, however, for this proposal is the experimental demonstration of single-donor placement within clusters with atomic precision accuracy, allowing the lowest error rates. To date the highest accuracy that has been achieved is \pm 1 lattice spacing $^{23}$, and work is currently underway to increase this.

In summary, we have demonstrated high-fidelity projective spin readout in a multi-P donor cluster, patterned in an atomically precise charge-sensing architecture. Importantly, we show long relaxation times ( $\approx$ seconds), comparable to that of a single $\mathrm{P}$ donor in silicon. Using tight-binding calculations to determine the difference in hyperfine coupling in donor clusters of different sizes, we demonstrate the applicability of these systems for qubit addressability in a globally applied magnetic field.

\section{Methods}

Device fabrication. The device was fabricated on the $(2 \times 1)$ reconstructed surface of a n-doped $(1-10 \Omega \mathrm{cm}) \mathrm{Si}(001)$ wafer, in an ultra-high-vacuum STM chamber. After removal of the native oxide, the surface was first passivated with a monolayer hydrogen mask, and then patterned with the STM tip (4-7 V, 5-10 nA) to create the device template (Fig. la,b). Subsequent exposure to $\mathrm{PH}_{3}$ gas and annealing at $350{ }^{\circ} \mathrm{C}$ for $1 \mathrm{~min}$ led to $\mathrm{P}$ incorporation, selectively within the device template. Once realized, the whole device was overgrown with $\approx 25$-nm epitaxial $\mathrm{Si}$ in the same chamber. Aluminium leads were then fabricated ex situ by standard electron beam lithography and metallization techniques, to make Ohmic contacts to the buried device.

Surface chemistry of $\mathbf{P}$ incorporation. Within the lithographic pattern of $\mathrm{D}_{2}$ (Fig. 1c-e), only the two rows of five Si dimers (marked by green ellipses) can incorporate $\mathrm{P}$ donors, while the rest (in yellow) cannot. This is understood by considering the series of dissociative surface chemical processes, which leads to $\mathrm{P}$ incorporation in silicon from the gaseous $\mathrm{PH}_{3}$ precursor ${ }^{11}$. The schematics of Supplementary Fig. S3 illustrate these processes. Dosing at sufficiently high rate leads to dissociation of $\mathrm{PH}_{3}$ molecules into $\mathrm{PH}_{2}+\mathrm{H}$, at every exposed $\mathrm{Si}$ dimer site (Supplementary Fig. S3a). This inhibits any further reaction at room temperature. However, when the surface is subsequently heated up to $350{ }^{\circ} \mathrm{C}$ for annealing, one $\mathrm{PH}_{2}$ fragment recombines with a $\mathrm{H}$ atom and desorbs as $\mathrm{PH}_{3}$ (Supplementary Fig $\mathrm{S} 3 \mathrm{~b})$. This results in one dimer site being available for immediate dissociation of another $\mathrm{PH}_{2}$ to $\mathrm{PH}+\mathrm{H}$ (Supplementary Fig. S3c). Finally, the third $\mathrm{PH}_{2}$ desorbs as well, making room for further dissociation of the $\mathrm{PH}$ to $\mathrm{P}+\mathrm{H}$ (Supplementary 
Fig. S3d). Still under annealing conditions, the $\mathrm{P}$ atom exchanges site with a $\mathrm{Si}$ atom of the top layer, thereby being incorporated (Supplementary Fig. S3e). As evident from the schematic diagrams, five bare-silicon sites (or 2.5 adjacent $\mathrm{Si}$ dimers) are required to incorporate a P donor. This is only satisfied within the two rows of five Si dimers in Fig. 1c-e, and makes the most likely donor count of $\mathrm{D}_{2}$ to be four. On the basis of the understanding of the dissociative surface chemical processes, it is also evident that the five dimer patches of Fig. 4 can host a maximum of two $\mathrm{P}$ donors.

Electrical measurements. For all electrical measurements, the device was mounted on a high-frequency printed circuit board within a copper enclosure, thermally anchored to the cold finger of a dilution refrigerator, with a base temperature of $\approx 100 \mathrm{mK}$. Direct current voltage was applied to the two gates and added to the voltage pulses generated by an arbitrary waveform generator. The SET current, $I_{\mathrm{SD}}$, was amplified, converted into a voltage signal at room temperature, filtered with an 8th order low-pass Bessel filter, and measured with a fast digitizing oscilloscope.

Calculation of hyperfine coupling. The strength of hyperfine coupling, $A$, is proportional to the probability density $\left|\Psi\left(r_{i}\right)\right|^{2}$ of the electron wavefunction at the site of the donor atom $r_{i}$ (ref. 19). An applied electric field $(E)$ distorts the wavefunction and, therefore, modifies $A$. To calculate the $E$ dependence of $A$, the electron wavefunctions were first obtained from the semi-empirical tight-binding method in the Nanoelectronic Modeling tool (NEMO-3D), as described in ref. 19. In addition to the contact hyperfine components $A$, we also calculate the anisotropic components of the full hyperfine tensor $\mathbf{B}$ (as described in ref. 24) to estimate whether the modified wavefunction of an electron bound to a donor cluster poses an obstacle to the coherent manipulation of the electron spin. We found for the 2-P cluster depicted in Fig. $4 \mathrm{a}\left|B_{\mathrm{xy}}\right|=89.9 \mathrm{kHz}$,

$\left|B_{\mathrm{xz}}\right|=52.3 \mathrm{kHz},\left|B_{\mathrm{yz}}\right|=15.5 \mathrm{kHz}$, all approximately 0.001 times the contact hyperfine term. The tight-binding method is an atomistic full band method that effectively captures the multi-valley structure and valley-orbit interactions present in silicon, providing a highly accurate description of Stark-shifted single-donor states. The method has been very effective in explaining a variety of donor experiments in silicon ${ }^{23,25}$

\section{References}

1. Tyryshkin, A. M. et al. Electron spin coherence exceeding seconds in highpurity silicon. Nat. Mater. 11, 143-147 (2012).

2. Witzel, W. M., Carroll, M. S., Morello, A. M., Cywinski, L. \& Das Sarma, S. Electron spin decoherence in isotope-enriched silicon. Phys. Rev. Lett. 105, 187602 (2010)

3. Steger, M. et al. Quantum Information storage for over $180 \mathrm{~s}$ using donor spins in a ${ }^{28} \mathrm{Si}$ 'semiconductor vacuum'. Science 336, 1280-1283 (2012).

4. Feher, G. \& Gere, E. A. Electron spin resonance experiments on donors in silicon. II. Electron spin relaxation effects. Phys. Rev. 114, 1245-1256 (1959).

5. Castner, Jr T. G. Direct measurement of the valley-orbit splitting of shallow donors in silicon. Phys. Rev. Lett. 8, 13-15 (1962).

6. Morello, A. et al. Single-shot read-out of an electron spin in silicon. Nature 467, 687-691 (2010).

7. Hollenberg, L. C. L., Greentree, A. D., Fowler, A. G. \& Wellard, C. J. Twodimensional architectures for donor-based quantum computing. Phys. Rev. B 74, 045311 (2006)

8. Morton, J. J. L., McCamey, D. R., Eriksson, M. A. \& Lyon, S. A. Embracing the quantum limit in silicon computing. Nature 479, 345-353 (2011).

9. Pla, J. J. et al. A single-atom electron spin qubit in silicon. Nature $\mathbf{4 8 9}, 541-545$ (2012).

10. Mahapatra, S., Büch, H. \& Simmons, M. Y. Charge sensing of precisely positioned P donors in Si. Nano Lett. 11, 4376-4381 (2011).

11. Wilson, H. F. et al. Thermal dissociation and desorption of $\mathrm{PH}_{3}$ on $\mathrm{Si}(001)$ : a reinterpretation of spectroscopic data. Phys. Rev. B 74, 195310 (2006)

12. Morello, A. et al. Architecture for high-sensitivity single-shot read-out and control of the electron spin of individual donors in silicon. Phys. Rev. B 80, 081307 R (2009).
13. Elzerman, J. M., Hanson, R., Willems van Beveren, L. H., Witkamp, B., Vandersypen, L. M. K. \& Kouwenhoven, L. P. Single-shot read-out of an individual electron spin in a quantum dot. Nature 430, 431-435 (2004).

14. Hasegawa, H. Spin-lattice relaxation of shallow donor states in Ge and Si through a direct phonon process. Phys. Rev. 118, 1523-1534 (1960).

15. Thewalt, M. L. W. Details of the structure of bound excitons and bound multiexciton complexes in Si. Can. J. Phys. 55, 1463-1480 (1977).

16. Mayur, A. J., Sciacca, M., Ramdas, A. K. \& Rodriguez, S. Redetermination of the valley-orbit (chemical) splitting of the 1 s ground state of group- $\mathrm{V}$ donors in silicon. Phys. Rev. B 48, 10893 (1993).

17. Hill, C. D., Hollenberg, L. C. L., Fowler, A. G., Wellard, C. J., Greentree, A. D. \& Goan, H.-S. Global control and fast solid-state donor electron spin quantum computing. Phys. Rev. B 72, 045350 (2005).

18. Bradbury, F. R., Tyryshkin, A. M., Sabouret, G., Bokor, J., Schenkel, T. \& Lyon, S. A. Stark tuning of donor electron spins in silicon. Phys. Rev. Lett. 97, 176404 (2006).

19. Rahman, R. et al. High precision quantum control of single donor spins in silicon. Phys. Rev. Lett. 99, 036403 (2007).

20. Rahman, R., Park, S. H., Boykin, T. B., Klimeck, G., Rogge, S. \& Hollenberg, L. C. L. Gate-induced g-factor control and dimensional transition for donors in multivalley semiconductors. Phys. Rev. B 80, 155301 (2009).

21. Martins, A. S., Capaz, R. B. \& Koiller, B. Electric-field control and adiabatic evolution of shallow donor impurities in silicon. Phys. Rev. B 69, 085320 (2004).

22. Kane, B. E. A silicon-based nuclear spin quantum computer. Nature 393, 133-137 (1998)

23. Fuechsle, M. et al. A single atom transistor. Nat. Nanotech. 7, 242-246 (2012)

24. Park, S. H., Rahman, R., Klimeck, G. \& Hollenberg, L. C. L. Mapping donor electron wave function deformations at a sub-Bohr orbit resolution. Phys. Rev. Lett. 103, 106802 (2009).

25. Weber, B. et al. Ohm's law survives to the atomic scale. Science 335, 64-67 (2012).

\section{Acknowledgements}

We thank R. P. Starrett and D. Barber for technical assistance and acknowledge support of the Australian Research Council Centre of Excellence for Quantum Computation and Communication Technology (project number CE110001027), the US national Security Agency and the US Army Research Office under contract number W911NF-13-1-0024. M.Y.S. acknowledges a Federation fellowship. R.R. thanks G. Klimeck for the NEMO-3D tool and acknowledges support of Sandia National Laboratories, which is a multi-program laboratory managed and operated by Sandia Corporation, a wholly owned subsidiary of Lockheed Martin Corporation, for the U.S. Department of Energy's National Nuclear Security Administration under contract DE-AC04-94AL85000.

\section{Author contributions}

S.M. designed the device layout and fabricated it with H.B. H.B. performed the measurements with inputs from A.M., S.M. and M.Y.S. R.R. carried out the tight-binding calculations. H.B., S.M. and A.M. analysed the readout data, and S.M., R.R., H.B. and A.M. analysed the modelling results. M.Y.S. planned the project. S.M. and H.B. wrote the manuscript, with inputs from all co-authors.

\section{Additional information}

Supplementary Information accompanies this paper at http://www.nature.com/ naturecommunications

Competing financial interests: The authors declare no competing financial interests.

Reprints and permission information is available online at http://npg.nature.com/ reprintsandpermissions/

How to cite this article: Büch, H. et al. Spin readout and addressability of phosphorusdonor clusters in silicon. Nat. Commun. 4:2017 doi: 10.1038/ncomms3017 (2013). 